\title{
Fidelity First in Middle School Reading Programs
}

\author{
Emir Gonzalez ${ }^{1}$, Michelle McCraney ${ }^{1,2}$, Sunddip Panesar-Aguilar ${ }^{1,2, *} \&$ Chri Cale $^{1}$ \\ ${ }^{1}$ School of Education, Walden University, Minneapolis, Minnesota, United States \\ ${ }^{2}$ College of Health Sciences, University of St. Augustine, Florida, United States \\ *Correspondence: College of Health Sciences, University of St. Augustine, St. Augustine, FL, United States. Tel: 1- \\ 904-770-3676. E-mail: saguilar@usa.edu
}

Received: February 19, 2020

Accepted: April 8, $2020 \quad$ Online Published: April 20, 2020

doi:10.5430/wje.v10n2p109

URL: https://doi.org/10.5430/wje.v10n2p109

\begin{abstract}
Middle school reading scores throughout the state of California have been predominantly less than average in recent years. A school located within this region has struggled to raise reading scores. An unknown problem existed that stemmed from the implementation of the school's reading program. The purpose of this investigation was to (a) determine the level of fidelity to the reading program, (b) understand the teachers' perceptions of the reading program, and (c) understand the structure of the reading program. The theory of andragogy guided this qualitative case study. Six teachers from a local school participated in the investigation. The teachers were purposely selected to take part in semi-structured interviews. Two sets of data were gathered for this investigation: (a) results from semi-structured interviews, and (b) publicly available reading data. The data were coded, and emerging themes were outlined. Six themes emerged to understand the overall process of the reading program. The results of the study pointed to the need for a more focused and sustained reading program. Another finding from the investigation was that teachers need year-around training in implementation fidelity. Another finding was that the reading program's structure can benefit from the 5 constructs that make up implementation fidelity. The implications of this study may affect positive social change by providing teachers with sustained training and support to be effective reading development facilitators. Well-trained teachers have a profound effect on their students and providing teachers a platform to guide these students toward a literate world can make a positive social change in their communities.
\end{abstract}

Keywords: fidelity, reading program, literacy, reading

\section{Introduction}

\subsection{Introduce the Problem}

Closing the reading gap among students with below-basic comprehension skills in schools throughout the United States is a challenge not only for schools and districts, but also for political and economic entities (Moore, Gove, \& Tietjen, 2017). Schools face the challenge of educating students with an array of learning difficulties, socioeconomic backgrounds, and learning abilities. The cost of improving one student's reading ability through academic intervention is $\$ 25,000$ per year (Moore et al., 2017). Budgetary issues arise every school year and lead schools to abandon the reading program. The money allocated to schools is used for teaching personnel, materials, and the purchase of expensive reading programs. Also, training teachers can be costly. However, for an intervention to be effective, teachers need to be prepared to change their practices and to be able to implement research-based interventions. Educational leaders must be strategically committed to implementing an effective reading program. Moore et al. (2017) stated, "The current educational system is no accident, and political powers need to be controlled by all educational stakeholders" (p. 14). Despite problems that educators face, it is paramount that stakeholders work together to increase student achievement.

In the United States (U.S.), two in three students are not reading at grade level and need intervention. For decades, students are being promoted to the next grade and schools are ignoring the reading problem. Also, as students are promoted, little intervention is taking place at the school level, causing the reading problem to compound. Intervention is given to those students who are failing an English class, have not reclassified, and who score below proficiency in the Smarter Balance Test (L. Hernandez, Personal Communication, February 27, 2018). Intervention must be targeted and specific to the needs of the intervention group. According to O'Reilly, Weeks, Sabatini, 
Halderman, and Steinberg (2014), "Many literacy intervention programs fail to demonstrate reading improvement in students, and they suggest that a misalignment exists between the needs of students and the goals of the intervention" (p. 405). Despite the obvious misalignment, schools continue to struggle to create effective intervention programs. Schools leaders have the right intentions, but they often face challenges during the delivery of the intervention reading programs. These challenges include, but are not limited to, time allotted for the intervention, lack of resources, different methods of delivery by teachers, and a teacher's experience. The challenges that schools face can be curtailed with the right teacher training program.

Another possible explanation for the gap in practice in reading intervention is the inadequate support and training teachers receive (Polkinghorne, 2013). In RST School District (RSTSD, a pseudonym), the reading gap exists because the current reading curricula does not meet the needs of a diverse student population in reading. Exacerbating the problem, there is a lack of research existing that demonstrates the direct relationship between the reading curriculum and student achievement. In this study, the researcher focused on the insufficient amount of literature on fidelity of implementation (FOI). If teachers or educators want reading programs to be successful, further research on the five constructs of FOI needs to be conducted (Van Kuijk, Deunk, Bosker, \& Ritzema, 2015). The term FOI is investigated through a different lens by Dane and Schneider (1998). FOI includes five critical elements: adherence, dosage, quality of instruction, participant responsiveness, and differentiation (Dane \& Schneider, 1998). Dane and Schneider focused on providing a comprehensive approach to implementing a program. Dane and Schneider revealed the need to examine the implementation of innovating programs through the five constructs.

Literature indicates that most of the research conducted on FOI is based on one or two elements of FOI. However, limited time is spent on examining a program through the lens of all five elements of FOI. Research limitations on FOI suggest that possible reasons that intervention programs fall short in reaching their goals is due to the lack of program loyalty. FOI is defined as the extent to which an intended program is implemented and leads to a positive outcome in students (Guo et al., 2016). The extent to which an intervention program is implemented is influenced by measuring the following variables: adherence, dosage, quality of instruction, participant response, and curricular differentiation. The mentioned variables, if measured accurately, can lead to student achievement in the classroom. When measuring adherence, the goal is to examine teacher self-reports about the activities and methods in which the program is delivered. This approach allows the researcher to compare what is expected from the teacher with what is taking place. The self-report allows the teacher to examine his or her own level of adherence, giving the teacher the opportunity to make necessary adjustment, to ensure proper adherence. When dose is measured effectively, the investigator considers logs, checklists, and self-reports as important tools. A checklist keeps the teacher on pace to deliver the intended program. School staff needs to understand that there is no teacher-proof curriculum that will lead to the ideal results; however, the quality of instruction increases with the proper teacher training and support. Teachers who are highly trained and supported will be more responsive to the program. These constructs are divided into the structure and process of a reading program. Measuring structure and process separately allowed me to organize and separate by data into distinct categories. Literature has suggested that adherence and dosage are the most important elements of FOI. For this reason, dosage and adherence was used to provide the overall structure of the study. Quality of instruction, differentiation, and teacher responsiveness make up the process of the study. The justification for assigning the three elements to the process is due to the ease in which the three constructs can be analyzed during the implementation process. In Table 1, the five FOI constructs are separated into structure and process. The structure consists of adherence and dosage and the other three constructs (quality of instruction, differentiation, and teacher responsiveness) are organized into the process.

Table 1. Segregation of Components of Fidelity of Implementation into Structure and Process

\begin{tabular}{lc}
\hline Components & Constructs of fidelity of implementation \\
\hline Structure & Adherence and dosage \\
Process & Quality of instruction, differentiation, and teacher responsiveness \\
\hline
\end{tabular}

As school leaders attempt to increase student achievement through reading programs, leaders must analyze how a program is implemented. School leaders must determine the most efficient way to implement literacy programs with fidelity. Moreover, school leaders must find effective means to measure the five elements of fidelity. To mitigate the reading dilemma in academia, it is necessary to examine the ways that literacy programs are implemented with fidelity. 


\subsection{Explore Importance of the Problem}

The problem is that the extent to which a reading program is being implemented with fidelity at Gamma School (GS, a pseudonym) is unknown. GS is one of several schools struggling to meet reading standards at the RSTD. The RSTSD ranks in the bottom one-third of school districts in reading (California Assessment of Student Performance and Progress [CASPP], (2018b). RSTSD Smarter Balance Assessment (SBAC) results are 10\% lower compared with the state results (CASPP, 2018a). The 10\% difference in reading is based on 8th-grade students who took the SBAC in 2017. The district is trying to improve reading scores by directing monies toward reading intervention curriculum. The high number of students who fail to read proficiently continues to increase and has become a pressing issue for our schools that requires immediate attention (Fogarty, Oslund, Simmons, Davis, Simmons, Anderson, Clemens, \& Roberts, 2014). RSTSD has implemented after-school reading intervention programs to improve reading scores. One-hour sessions are taking place 2 days a week for 10 weeks, to help improve reading proficiency. The entire time used to teach the reading curriculum focuses on teaching reading skills by using various reading strategies. Moore et al. (2017) indicated that frequency, intensity, and fidelity influence the overall expected outcome of a reading program. These elements mentioned lead to a focused and targeted curriculum for struggling school-aged students. Literature has suggested that frequency and intensity can be part of the FOI (De LA Paz et al., 2014; Fogerty et al., 2014). Intensity and frequency often fall into the construct of dosage, and dosage is part of FOI.

In this study, FOI of a reading program that encompass the reading curriculum at GS was investigated. The focus in this study was to examine the five elements of FOI (adherence, dosage, quality of instruction, teacher responsiveness, and differentiation). The examination is mitigated by the categorization of the five components of FOI into structure and process (see Table 1). To be effective, a reading curriculum must focus on every aspect of FOI (Fogarty et al., 2014). School leaders need to do a better job of incorporating all aspects of FOI. Schools perceive fidelity as adherence to the instructional plan. However, the complexity of all five elements makes adhering to the plan difficult, especially if each element is not categorized and considered individually. Therefore, the aforementioned elements mentioned are crucial to the FOI of a curriculum.

RSTSD uses a variety of reading programs. The process by which the school district selects its reading curriculum is unknown. However, evidence-based reading programs are being discussed. Evidence based indicates that the selected programs have been researched and validated. One example of a reading program is the Intensive Diagnostic Educational Center (IDEC). IDEC is an evidence-based approach to reading that the RSTSD has implemented throughout its schools. Teachers are selected and trained to work with struggling readers. The district selects English teachers working with students who struggle with literacy.

In this study, reading programs, through the lens of a teacher and their role in the process and structure of the reading program GS has implemented, were investigated. Segregating the process and structure of the program allows the researcher to categorize and analyze each element of FOI. De La Paz et al. (2014) suggested that students tend to score higher on reading assessments when teachers implement reading intervention curriculum with fidelity. To see the effectiveness of a reading program, the researcher explored the five components of FOI. Emphasis on how well teachers at GS used the five elements of FOI were explored.

In the RSTSD, little is known about reading programs and FOI. Schools are implementing reading programs, but there are obvious gaps in the implementation process. Program facilitators should consider the needs of students, flexibility of curriculum, adaptive planning process, relevant training, and continuous monitoring. More needs to be done to improve the effectiveness of reading intervention curriculum in the RSTSD. RSTSD schools are implementing the reading curriculum programs but are not obtaining the desired results. Perhaps schools need to focus on encouraging teachers to be involved in the development of the reading programs. If the planning, implementation, and monitoring of the reading program are in synchrony, the likelihood of reaching the desired results increase. The overall goal of a program will be attainable for all students, providing them with the opportunity to perform at grade level during the end of the year summative assessments.

GS is among the lowest performing schools in RSTSD. Almost three-quarters of GS 7th- and 8th-grade students did not meet the ELA standards on the SBAC in 2017 (CASPP, 2018b). These numbers are consistent throughout middle schools in the RSTSD. In reading, $8 \%$ of 7 th graders scored above standard, and $11 \%$ of 8 th graders scored above standard in 2017 (CASPP, 2018b). In research/inquiry, 11\% of 7th- and 8th-graders scored above standard on the SBAC (CASPP, 2018b). The overall mean score for both groups is 2480 , indicating that most students fall in the range of achievement level scale score for standard not met (CASPP, 2018b). See Table 2. 
Table 2. RSTSD 7th- and 8th-Grade SBAC Performance in English Language Arts, 2017

\begin{tabular}{lccc}
\hline Grade & $\begin{array}{c}\text { Percentage proficient in } \\
\text { SBAC reading }\end{array}$ & $\begin{array}{c}\text { Percentage proficient in } \\
\text { research \& inquiry }\end{array}$ & Overall mean score \\
\hline $7^{\text {th }}$ & $8 \%$ & $11 \%$ & 2480 \\
$8^{\text {th }}$ & $11 \%$ & $11 \%$ & 2480 \\
\hline
\end{tabular}

Note. California Assessment of Student Performance and Progress (2018a; 2018b).

The purpose of this qualitative instrumental case study study was to investigate implementation fidelity of the different reading programs at GS. The implementation process includes but is not limited to the strategic development of the curriculum in the program, curriculum alignment, the use of data, training, reading strategies, and teaching models. Current data reveals that students at GS are struggling to read at grade level. Although GS has a reading intervention program curriculum, students are not performing well in yearly standardized tests. A qualitative case study was conducted to investigate the current problem at GS. The case study consisted of interviews, archival data, and standardized test scores to determine the effectiveness of the ongoing implementation process of the reading intervention curriculum.

\subsection{Relevant Literature}

\subsubsection{Conceptual Framework}

The study is grounded on the Malcolm Knowles theory of adult learners. Andragogy has been around for 150 years (Taylor \& Kroth, 2009). However, it was Malcolm Knowles who dedicated much of his time to define the term Andragogy. According to Knowles (1984) adult learners are more likely to succeed in a learning process (implementation of a reading program) if the following principles are in place: A high level of transferability, involving the adult learner in the process, making the process relevant to the learners' life, utilizing the learners' prior knowledge, and making the process problem centered. The easier it is for an adult learner to transfer the new learning experience into the classroom the more successful the adult learner becomes. Also, if the adult learner is involved in his/her learning and the learning is relevant, the chances of success increase for that learner. Lastly, making the learning experience problem centered allows adult learners to be more interested and committed to the learning process. According to Knowles (1984) adult learners are naturally eager to take on a learning challenge. Creating a learning experience that is challenging can motivate an adult learner to persevere.

In a study conducted by Lambert, Gallagher, and Abbott-Shim (2015) their focus was to evaluate a mentoring program's FOI via the theory of andragogy. Using the five constructs of FOI (adherence, dosage, quality, differentiation, and responsiveness) Lambert et al. examined the level to which a mentoring program was delivered as intended. A simplistic fidelity checklist was used to determine the level of implementation fidelity. The fidelity checklist uses a plus and minus rating to determine IF. In their study the instrument focused on dosage and adherence. A limited amount of data was presented regarding quality, responsiveness and differentiation. Lambert et al. (2015) indicate that through andragogy they implemented coaching, observations, and reflective practices. The three mentioned implementations will be part of the proposed workshop. Lambert et al. concluded that using andragogy and FOI dramatically increase teacher performance and student achievement. Giving adults the opportunity to learn under certain conditions (applying the five principles) can excel in the learning process.

\subsubsection{Rationale}

Investigative findings suggest that teachers at GS need more training and support as they deliver their reading program. Although there is a need for teacher training and support, the school is not the only campus in the nation with the aforementioned problem. Evidence points to the lack of confidence by educators when implementing evidence-based practices (Lambert et al., 2015). These findings are in conjunction to findings from the present investigations. That is, teachers at GS lost confidence during the implementation process of their current reading program due to the lack of training. Empirical evidence consistently points to the need for training as a major barrier to the improvement of schools (Brock \& Carter, 2017). Teachers at GS did not have the opportunity to attend workshops and become familiar with the reading program expectations. Researchers are expressing concern about the overall implementation process of any program (Brock \& Carter, 2017). There are many approaches to the effectiveness of an implementation process.

The approach the researcher am suggesting is one of several different approaches to an effective implementation process that ensures fidelity. The researcher am proposing an implementation process that considers learning 
outcomes, reading expectations and the five constructs of IF. Moreover, connecting the mentioned components using an IF checklist. Teachers are the most important piece of the implementation process (Vollmer, Gettinger, \& Begeny, 2019). Teachers are the ones who will deliver the program and should be confident and prepared to deliver a reading program with high fidelity. The potential to decrease the reading gap via implementation fidelity is exponential. A single plan workshop must be delivered and supported throughout the year. A single plan workshop in this context is a workshop that is tailored to meet the needs of the school. A recommendation made by Buthane (2017) is to incorporate teacher training that covers system changes, principles of management, application of research-validated instruction, and management practices.

The core concepts mentioned provides direction and autonothe to a teacher delivering a reading program. Providing training on principles of management gives teachers the ability to take control of what is going on in the classroom. The idea that teachers become managers/facilitators leads to a student-centered classroom. Bethane (2017) attributes a successful implementation process to high levels of fidelity. She goes on to say that utilizing a fidelity checklist will improve fidelity level and success rate of the program. Literature throughout academia continues to stress the importance of high accuracy implementation. The more targeted a teacher training program is, the higher the chances of aligning the core components of a reading program to the expected outcome (Sun \& De La Rosa, 2015). Aligning core components of a program can include aligning student learning outcomes (SLO) to assessment protocols. Often a disconnect exist between what is expected from the learner, and how the learner is being assessed. This phenomenon becomes important because schools do not score proficient in end of the year summative assessments. One can argue that the program did not work and blame everyone involved. However, the lack of implementation fidelity could have contributed to the lack of success. Programs are set up to succeed but often they fail. Leaders must investigate every aspect of the program before they decide. The next section of the review of literature examines incorporating student learning outcomes into a reading program to ensure assessment alignment.

\subsubsection{Student Learning Outcomes (SLO) and Learning Targets}

A study conducted by Kim et al. (2016) points to the need for alignment of the curriculum to assessment outcomes (p. 365). Teachers and students are given the daunting task to perform at high levels of expectation using an unaligned process. Teachers are told to deliver a reading program with minimal training and students are expected to reach proficiency with an unaligned curriculum.

Teachers and students must be aware of the learning outcomes before a program is delivered. According to Kratz et al. (2019), the higher the implementation climate, the greater the student outcome becomes. In this context implementation climate refers to the extent to which use of an intervention is expected, supported, and rewarded. That is, curricular awareness increases the overall success of the program. Also, the implementation climate and implementation fidelity are directly related because both have a set of expectations. Implementation climate can be set in the classroom through professional development or teacher training. The teacher training program can outline the program's expectations and help teachers monitor their own progress and student progress. Student learning outcome can be enhanced by proving a reading program that is relevant, accessible, engaging and cognitively challenging (Kim et al., 2016). A reading program that is engaging provides students with the opportunity to practice in the classroom. An engaging classroom is a classroom with a high level of confidence. An accessible reading program aligns a learner's reading ability with Lexile level. Differentiating instruction for students provides a steppingstone for students to reach proficiency.

Kim et al. (2016) use a reading intervention program to align student learning outcomes with assessment via the Strategic Adolescent Reading Intervention (STARI). The goal of STARI is to promote high reading engagement that leads to reading achievement. Modes of assessment at times are not in conjunction to the reading program expectations. The simple fact that a student seems to be engaged does not guarantee that the student is engaged (Kim et al., 2016). STARI provides that engagement of element of learning in the classroom. The use of STARI provides real time practice for students to hone their reading time. An example utilized during a reading engagement lesson is the use of peer reading. The learning outcome is to interpret words and phrases that are used in the text. Together (with a partner) students take turns reading pieces of a text. Then, each student fills out an activity sheet that clarify words or phrases, students discuss and elaborate on parts of the text using quotes. The skills that students are developing are directly related to summative, end of the year testing concepts. Teachers play an important role in STARI because they can design lessons that are directly related to the program's expectation. To be able to reach expectations teachers are provided with a three-day workshop that addressed traits of struggling readings (Kim et al., 2016). Teachers participating in STARI had to attend the three-day workshop. What makes Kim et al. research applicable to the current project is that teachers attended a three-day workshop that included implementation fidelity. 
In a similar investigation, Andrews-Larson, Wilson, and Larbi-Cherif (2017) explore the concept of teacher collaborative time (TCT). The focus of Andrew-Larson et al. was to examine how structured, content-focused discussions improve instructional quality. The term structured in this context refers to limiting the ways teachers have discussions among each other regarding the learning process that takes place in the classroom. Buthane (2017) also suggests maximizing structure as a common approach to the demonstration of evidence-based classroom practice. A structured environment can lead to higher student achievement, positive academic outcomes and high levels of student engagement.

To improve the quality of instruction educators must examine the overall needs of the school. According to Andrews-Larson et al. (2017) "We seek to better understand the way in which teachers' collaborative conversations might support their ambitious teaching practice" (p. 4). Collaborative conversations provide the possibilities of a high level of teacher support, relevant day to day teacher talk, and alignment of curriculum materials and instructional goals for students. The key point here is the alignment of curriculum with student learning outcomes. Conversations must take place in the classroom, and in training sessions to get meaningful conversations. Teacher support in and out of the classroom can be demonstrated in different manners. In Andrews-Larson et al. study, the use of trained facilitators is used to provide sustainable support throughout the year. Facilitators need training that will help them successfully support teachers. Providing facilitators to a school can be costly and if not implemented correctly can be a waste of time. Andrews-Larson et al. suggest that facilitators must possess more knowledge than the teachers. Facilitator expertise is consequential for the potential to support teacher learning (Andrews-Larson et al., 2017 , p. 7). A productive facilitator can press teachers to develop meaningful and relevant conversations about learning. Also, facilitators establish clear goals aligned to learning outcomes, and assessments and identify the purpose behind each learning target.

Mediating teacher discussions can lead to positive learning outcomes for students and teachers. This concept becomes important to the study because it provides an opportunity to incorporate teacher collaborative time in the workshop. To optimize the efficiency of each workshop; time is needed to discuss best practices in a structured and rehearsed manner. Teacher collaborative time can be used to align student learning outcomes to assessments. Using the technique "facilitator pressed" Andrews-Larson et al. (2017) specific learning targets can be developed. In facilitator press the facilitator elicits explanations for each step of the learning target development. The facilitator requests an explanation from the teacher for choosing the learning target and providing a rationale for the alignment of an assessment to a specific learning target. Teacher involvement and responsiveness are key points in the investigation.

Teacher involvement and teacher responsiveness does not happen spontaneously. There must be buy-in from teachers to raise the level of responsiveness and involvement. Uzair-ul-Hassan, Parveen, and Riaz (2016) suggests creating a workshop that requires active learning from teachers (p.16). Active learning is driven by the accomplishment of goals a program sets on teachers. Active learning provides teachers an opportunity to actively get involved in the process and can be used as a model for teachers to practice. Engaging teachers in collaborative training can raise the quality of teacher performance and can raise student achievement (Van Kuijk et al., 2015). Teachers can get real time experience on their own learning style that can transferred into the classroom.

Providing a workshop that is participant centered will be an effective way to get teachers to respond in a positive manner and be more comfortable with the expectations of the workshop. According to Knowles principles of andragogy three of the five principles can be applied in active learning (transferability, the use of prior knowledge, and involvement). Knowles (1984) indicates that educators can tailor learning for adults through interactions, discussion forums that lead to problem solving, and solutions that can be immediately used in the lives of adult learners. In the investigation findings suggests that teachers want to do well, they are in the profession to help others and feel a sense of accomplishment. A workshop that can address teacher concerns with viable and research-based tools can lead to student and teacher success in the classroom.

\subsubsection{Assessment Cycle}

Another concern at GS is the deviation from the intended programs expectations. Teachers at GS implemented a reading program with a lack of uniformity. To develop a program that will address this concern literature was reviewed on implementation fidelity assessment. According to Vollmer et al. (2019) to ensure teachers are implementing the intended program with high fidelity data must be collected to assess the level of fidelity. Data collection on fidelity can be used to modify the curriculum without jeopardizing the integrity of the reading program. Also, assessing implementation fidelity allows teachers to understand the reasons why a program succeeded or failed. Smith et al. suggest integrating fidelity data with student learning outcomes and using assessment data to understand 
the unknown features that lead to the failure of a reading program. Unknown features in this context are unexplained reasons why a program fails to deliver positive results. Some unknown variables can include modifications that alter the program's integrity, not completing the delivery of the program as it was intended, and the role a teacher plays during the implementation process.

The role a teacher plays during the implementation process often can be subjective, meaning that interpretation of their role is defined via an observation or performance by the student in the classroom. To avoid misconceptions, the use of an implementation fidelity checklist/chart can help determine the role a teacher plays in the implementation process. In a fidelity checklist teachers and school staff can evaluate a teacher's performance during the implementation process. In a study conducted by Little, Riggs, Shin, Tate, and Pentz (2015) the use of an implementation fidelity chart was used to determine teacher participation and quality of instruction. The results of the study indicate that teachers who score higher on the fidelity chart did not deviate from the intended implementation of the program. On average teachers who participated in the implementation process delivered a higher quality of teaching and the result was a higher student achievement. Little et al. (2015) suggest teacher and administration buy in to a program can drastically increase the fidelity to which a program is implemented. Although the statement is true about buy in from staff, teacher support and training is critical to FOI. Guo et al. (2016) recommended a collaborative approach to teacher training and incorporating effective components to the teacher training can raise the overall quality of delivery and responsiveness of a teacher. There are two important notes to justify; that is, the effective components of a reading program, and quality and responsiveness. Effective components indicate training and support be given to teachers; moreover, the training and support must be focused on key reading components (phonemic awareness, comprehension, phonics, fluency). The training that takes place will focus on planning, teaching and reflecting on ways to improve reading scores. The quality of the delivery and responsiveness of participants (teachers) are important because they are two key ingredients that make up FOI. For this reason, Furtak et al. study becomes important to note in this document.

A targeted workshop becomes important because knowing what changes are taking place in the intended program is largely due to the implementation of an assessment cycle (Buthane, 2017). Furthermore, an assessment cycle included aligning learning outcomes with an assessment component and the assessment can be any form of instrumentation. The purpose of an assessment is to collect data that can leads to effective modification to the curriculum; therefore, leads to higher program achievement. Assessment data can facilitate the program's outcome by pinpointing details of the program each step of the way. Assessment data is important because it helps educators determine whether they are accomplishing the desired learning outcomes, data helps determine the level at which students are performing, and to answer questions from stakeholders (Buthane, 2017). Successful analysis of assessment data can lead to a high levels of implementation fidelity. Understanding why a situation is happening can be accomplished via data collection. The data collection can then be used to carefully modify changes to the curriculum without jeopardizing the intent of the program. Also, ensuring the quality of the program is intact and maintaining the correct path towards meeting student learning outcomes. This same concept is suggested by Smith, Finney, and Fulcher (2019) that understanding why events are taking place is crucial in the FOI of a program. A program is written to be successful in an ideal environment but often the ideal environment does not exist. Therefore, data collection becomes paramount during the delivery of a reading program. The analysis of this data will provide a school the opportunity to make changes leading to ideal outcomes.

\subsubsection{Implementation Fidelity Checklist}

Learning targets, and assessment cycles are important to the implementation of a program. The two constructs can provide a roadmap for curriculum builders. Implementation fidelity can be ensured by using a checklist. A checklist brings a few important checkpoints to the implementation fidelity process. This section of the review of literature provides details about the benefits of a fidelity checklist. According to De La Paz et al. (2014), rarely is the alignment of the planned intervention and the implemented intervention assessed simultaneously. The entire review of literature up to this moment has mentioned the need to align a curriculum with the actual outcome of the program. A dearth of evidence prevails in academia regarding instrumentation used to assess implementation fidelity.

The proposed workshop will use a checklist developed by the Finney (2019) to share with teachers and staff at GS. Table 3 represents a fidelity checklist consists of several parts. 
Table 3. Implementation Fidelity Checklist

\begin{tabular}{|c|c|c|c|c|c|c|}
\hline \multirow[t]{4}{*}{ Objectives } & Dosage & Program & Specific & Adherence & Quality & Responsiveness \\
\hline & & Component & Features & Yes/No & $1=$ Low & $1=$ Low \\
\hline & & & & & $2=$ Medium & $2=$ Medium \\
\hline & & & & & $3=$ High & $3=$ High \\
\hline
\end{tabular}

Student Learning Outcome 1

Student Learning Outcome 2

The structure that Finney (2019) follow is a 7-columns checklist indicating the objective/learning outcome, program component, and the five FOI constructs (adherence, dosage, quality, differentiation, and responsiveness). The objective/learning outcome is what will be measured. The program component can be a content standard that is aligned to the learning outcome. The first FOI construct assigned in the table is dosage of the program component, the length of the component. Next, the table identifies the program feature. The program feature become the differentiation aspect of the program. The program feature focuses on what will make the program different from what it being used. Following the differentiation, the table identifies the adherence to the program feature. To measure adherence Finney (2019) recommend a yes and no respond to each program feature. The next column feature is quality rating of (1-5). Here the observer is rating each specific feature. Lastly, it is the level of responsiveness. Again, using a rating of (1-5) is used to measure the responsiveness of the participant. The mentioned features make-up a fidelity checklist.

Completing a fidelity checklist can be done by a program auditor, implementers or facilitator(s) and participants (Finney, 2019). A program auditor can be an outside source that a school hires or district sends to evaluate a program. A facilitator can be an administrator, coach or department leaders. Participants (teachers) can fill out their own fidelity checklist that can mitigate the cost of bringing outside personnel to assess implementation fidelity. In a study done by Hall and Chapman (2018) on implementation fidelity, results indicate that the lack of implementation fidelity was due to the lack of funds to train and bring experts to support their teachers during the delivery of the program. Major barriers of FOI include physical resources and time (Hall \& Chapman, 2018). Funding is an issue in every school but that should not deter schools from properly implementing a program with high fidelity.

To save money, schools can be creative during the implementation process. Hall and Chapman (2018) indicate that training should be the first step in the implementation process. Teachers are eager to learn and lead (Knowles, 1984) and can provide support for their colleagues. This method of involving teachers in the training and support is cost efficient and provide a high-level autonofor teachers and administrators.

The utilization of a checklist can provide data on fidelity, learning outcomes and assessments. According to Smith, Finney, and Fulcher (2019), "A fidelity checklist provides a systemic way to capture all five of the implementation fidelity aspects" (p. 265). A systematic approach means analyzing the data and concatenating it to learning outcomes/learning targets. Moreover, a checklist provides educators to pinpoint strengths and weaknesses of the implementation process. Furthermore, educators can make practical informed modifications without worrying about changing the intended program and teachers can share their best practices with their colleagues so that they can replicate or expand the scope of the intervention (Smith, Finney, \& Fulcher, 2019).

The opportunity to replicate the program and to be successful with a program, provides experiential evidence, validity, and reliability to a program. As a researcher set out to conduct experiments, the goal is to make it replicable. Using a fidelity checklist makes replication easier to accomplish. Kaimal and Jordan (2016) contribute three findings to the lack of implementation fidelity: First, the lack of organization by staff. Second, the lack of knowledge of FOI. Third, the instrumentation tools and rubrics are too cumbersome and confusing. During the investigation Kaimal and Jordan did not use a fidelity checklist because they utilized evaluation sheets, and logs of length and frequency. Using a fidelity checklist provides precision with the components of the program that are being implemented. The elements of FOI are meant to keep teachers and staff aligned with the intended program delivery. A fidelity checklist provides details and organization of the intended program. The result of Kaimal and Jordan study revealed difficulties in pinpointing the exact reason for the low level of implementation fidelity.

\subsubsection{Teacher Reflection}

Professional teaching programs are turning to the practice of reflection to determine what worked, what didn't work, and where change needs to be made. Reflecting becomes important in a growth mindset; therefore, teachers must be 
open to constructive criticism and administrators open to proving constructive criticism without being disdainful. Reflecting should always lead to change. Wlodarsky (2018) states that, "The process of change through reflection is, in the most profound and authentic sense, learning to be a leader of one's own professional and personal self' (p. 39). Understanding and accepting an event that took place (teaching moment) is only the starting point of that event. Being able to reflect about the teaching moment allows a teacher to celebrate positive outcomes and to make changes to events that did not go as planned. According to Wlodarsky (2018), an event path for professional growth is the event that is taking place followed by a cognitive process that leads to a changing point and lastly creates a new event. An event can be in the form of a reading lesson that took place in the classroom. Providing teachers time to explore a cognitive process, provides teachers with the opportunity to discover a problem that took place during the delivery of the lesson. Through careful data analysis of the problem a decision to change the behavior is made. The changing point is supported by evidence via journaling, logs, observations or reflective instrumentation tool. Based on data and discussion a new event can take place and can be directly related to teacher growth. The model provides an organizational framework that is useful for teacher training, professional growth and self-evaluation (Wlodarsky, 2018). Cognitive processing can become a school wide strategy that drives an intervention reading program because school leaders can link reflection and the reading curriculum.

Reflection is a strategy that must be manipulated to benefit a teacher's outcome. Suhrheinrich and Chan (2017) considered the use of microteaching as a method of teacher reflection, and constructive feedback. In microteaching, teachers videotape a lesson they are giving, and later review the video with their peers. This strategy provides teachers the opportunity to focus on teaching behaviors. Teaching behaviors are often bypassed during observations and teacher evaluations. Also, microteaching allows teachers to focus on a specific feature (differentiation) of a lesson. Specific feature is a term used in an IF checklist (it is part of the 5 constructs of IF). Moreover, microteaching provides teachers a method to increase their IF level by magnifying the specific feature that is being implemented.

Performance feedback provides teachers the opportunity to reflect on their teaching skills. Performance feedback is an ongoing process that coincides with the model presented in the previously section by Wlodarsky (2018). Therefore, performance feedback can be more effective during training and coaching process. The goal is to provide teachers a structured opportunity to reflect on their practice to increase IF.

Proving teachers, a chance to cognitively think of their skills, behaviors, and learning can be beneficial to student outcome. This process can take place through self-reflection. Self-reflection can manifest into a teacher's self-growth. Teacher learning is an active process that allow teachers to participate in learning activities that can result in new approaches to pedagogy and develop new knowledge (Suhrheinrich \& Chan, 2017). Teachers that experience learning through reflection allows them to become familiar with the content. Teachers can learn from teaching when given the time to reflect. Educators can log their daily events that take place in the classroom and can stimulate a teacher to change a teaching behavior that leads to greater student achievement in the classroom.

Teacher reflection is an effective tool that help teachers develop meaningful lesson plans which can lead to higher student motivation in the classroom (Suhrheinrich \& Chan, 2017). Reflection logs help teacher examine a lesson delivery with a more critical lens. Teachers can cross reference their lesson plan with their reflection logs. This method of collecting teaching data provides stakeholders with the opportunity to development new knowledge and increase teacher growth. Teachers want to deliver the best performance in the classroom daily, they genuinely want to make a difference in the lives of their students. Knowles (1984) indicates that adult learners (teachers) want and would like to help others and that is why they are eager to learn. Understanding this concept of adult learners should allow the proposed workshop to be an opportunity for teachers to learn and grow.

\section{Method}

The research method that the researcher used in this study was a qualitative instrumental case study was used for the study. Using an instrumental case study allowed the researcher to gain insight into the broader issue of reading in the United States. According to Lodico, Spaulding, and Voegtle (2010), “An instrumental case study is in-depth, but it studies the specifics of the participants or settings to gain insight on the broader issue" (p. 163). The widespread problem is concerning: Students are not reaching proficiency as they make their way into high school. Selecting teachers that are involved or have been involved in the reading program as participants in the investigation provided specific information to answer the research questions. Teachers provided in-depth details of the reading program and their role in the reading program. Moreover, the investigation examined the role that teachers play in the FOI of a reading program. Knowles theory of andragogy provides the framework for the role teachers play in the FOI. The FOI process is separated into two sections: the structure of the reading curriculum and the implementation process of 
the reading curriculum. The research questions are derived based on the structure and process during the implementation phase. Also, the structure and process guided the interviews and qualitative study. Lodico et al. (2010) indicate that qualitative research uses primary narrative or verbal methods such as interviews to collect and summarize data. Semi-structured interviews were the primary source of data in the investigation.

\subsection{Description}

Meriam and Tisdell (2016) referred to a case study, "As an in-depth description and analysis of a bounded system" (p. 37). In this study, the bounded system was GS. A qualitative case study was appropriate for the study because the researcher examined a group of teachers (case) and the FOI process of a reading curriculum. The purpose in this study was to explore (a) the structure and process of the reading program, (b) how teachers implement the reading program, and (c) teachers' perception of the reading curriculum at GS.

The goal was to identify 12 teachers. The email provided teachers with a summary of the investigation and procedures during the investigation. To ensure the safeguard of all participants, participants signed a letter of informed consent. Reading program teachers were sent another email. The email asked them to submit the letter of informed consent via email. The researcher picked up three informed consents from three participants. Obtaining informed consent and addressing possible concerns raised by participants were dealt with during the scheduled one on one interviews.

\subsection{Participant (Subject) Characteristics}

The participants in this study were middle school teachers who have been involved in the implementation process of a reading program. Teachers participating in this research were familiar with a reading program structured to facilitate the needs of struggling readers and had experience in the reading intervention curriculum. 12 teachers were invited to participate, and six teachers were ultimately recruited. The six teachers became a limitation to the investigation because the research has limited data, making it difficult to generalize the results.

\subsection{Sampling Procedures}

To select teachers, purposeful sampling was utilized. Purposeful sampling is a popular choice for a qualitative investigation (Creswell, 2012). Purposeful sampling allows a researcher to understand and gain insight into a phenomenon taking place in a setting. Therefore, the sample must be carefully selected to provide rich and meaningful data (Meriam \& Tisdell, 2016). The purpose in this investigation was to gain meaningful insight on FOI; therefore, meaningful data must come from experts (teachers) in the area of reading programs. The use of purposeful sampling allowed the researcher to deliberately choose the participants with experience in the implementation of a reading program. The criteria for the selection process was to communicate with the principal of GS. The goal was for the principal to direct the researcher to the assistant principal overseeing the English Department. In addition, the principal was asked to introduce the researcher to the reading program coordinator at the school site. Taking these steps allowed the researcher to purposefully select the teachers for this investigation.

Middle school teachers with experience in teaching a reading program were selected. The group of teachers chosen are from the same middle school and are teaching the reading program that was investigated. This approach makes the use of a purposeful sampling appropriate. The aim in the investigation was to select 12 reading recovery teachers. A conscious effort was made to ensure the number of participants did not fall below 12. According to Lodico et al. (2010), a "Homogeneous sample allows a researcher to work with a smaller population within a larger group of people" (p. 138). The key is to ensure that all participants share similar experiences or attributes of a reading program. A valid concern was that the sample size did not reach 12. A strategic plan was in place to gain access to the participants. Ethical considerations were considered and ensuring the safety of all parties in this investigation is paramount. To obtain access to the investigation, standard research procedures were followed.

\subsubsection{Research Design}

A semi-structured interview protocol was used to gather important data on FOI at Gamma School. To set the parameters of the semi-structured interview, an interview protocol was established. The goal of the interview protocol was to lay out the purpose of the interview, and the role the interviewee would play through the study. Also, the protocol reminded the interviewees why this research was being conducted. To validate parts of the interview district reading data found in the Department of Education database was used as a reference point, and to confirm what was said in the interviews. Also, the use of reading scores provided the researcher with important information regarding the overall reading achievement of GS. Furthermore, the examination of summative state literacy tests scores allowed the researcher to compare what was expected from students and the alignment of the reading curriculum. The intentions were not to quantitatively analyze district reading scores but rather, to get a better 
understanding of the overall reading achievement for the past three years at Gamma School. The interview questions stem from the three research questions. The interview questions provided ideal data for this research. The questions were aligned to the conceptual framework of Andragogy (Knowles, 1984).

\section{Results}

The reading gap in the U.S. is pervasive and is not going away anytime soon. GS is a school located in the western part of America that is facing a reading crisis. Low performing scores throughout the school is causing concern for all stakeholder at GS. The purpose of this study was to examine the level of FOI of the school's reading program. Data collection took place in three steps. The first step was to gain access to the school. Obtaining permission from the principal was the top priority of the investigation. An email was sent to the principal. In the email, the emphasis was introducing the purpose of the investigation and request permission to send emails to teachers at the school. The recruitment process was discrete, and confidentiality was applied to every part of the investigation. Once permission was granted, emails were sent to potential teacher participants. Teachers were responsive to the email; therefore, all six responded to the email. In the email teachers were given a summary of the investigation, and details were highlighted regarding the teacher's role in the investigation. Teachers responded within 48-hours, and interviews took place the following week. Before the interview process could begin a letter of cooperation was drafted and signed by the principal. Table 4 groups research questions, themes and codes together. Also, Table 4 links themes and codes to each of the three research questions.

Table 4. Themes and Codes Associated to Research Questions

\begin{tabular}{lll}
\hline Research question & Themes & Codes \\
\hline RQ1: (SQ 1-5) & Theme 1: Problems with program & Code 1: Lack of program direction, \\
How and in what ways are teachers & expectations (adherence) & $\begin{array}{l}\text { unclear teacher expectations, lack of } \\
\text { adherence documentation }\end{array}$
\end{tabular}

intervention curriculum at Gamma

School?

RQ1: (SQ 1-5)

How and in what ways are teachers implementing the reading intervention curriculum at GS?

RQ2: (SQ 6-13)

What constitutes the process of the reading intervention curriculum at GS?

\section{RQ2: (SQ 6-13)}

What constitutes the process of the reading intervention curriculum at GS?

RQ2: (SQ 6-13)

What constitutes the process of the reading intervention curriculum at GS?

RQ3: (SQ 14-15)

What are the teacher's perceptions of the reading curriculum at GS?
Theme 2: Insufficient amount of Code 2: Lack of teacher support dosage (teacher dosage) (2-hour training), and unaligned Professional development,

Theme 3: Different approaches to differentiation Aligning differentiation to expectations combining these two themes.

Theme 4: Issues with the pacing plan

Code 3: The reading program differentiates instruction

Code 4: Teachers using various methods of differentiation

Code 5: The need for more teacher training on differentiating instruction

Code 6: Struggles implementing a pacing plan

Code 7: Support in adjusting to pacing plan

Theme 5: Identifying the quality of instruction in the reading program

Code 8: Different perception to quality of instruction

Code 9: disconnect between reading expectation and quality of instruction

Theme 6: Willingness to a make a Code 10: Want to learn more about difference the reading programs and find ways to improve instruction 
Nvivo was utilized to confirm themes generated by the investigation. The open coding process resulted in ten codes and six themes. The themes mentioned are crucial to the investigation and provide a road map for next steps of this investigation. The findings are described according to themes and organized with respect to the question they answered. The findings are explained by Research Question.

\section{Research Question 1}

Research Question 1 asked the following: How and in what ways are teachers implementing the reading intervention curriculum at GS? Research question 1 is projected to determine the structure of the reading program. In this investigation, the structure of the reading program consists of adherence to the reading program, and the dosage teachers receive throughout the reading program. To answer research question 1, six questions were asked from the interview protocol. The opportunity to investigate to what extent are teachers delivering the reading program as intended (adherence) and the amount of training, coaching and support (dosage) teacher are receiving. Also, with what frequency are teachers receiving the dose. Two themes emerged from RQ 1.

\section{Theme 1: Problems with the Program's Expectations}

Theme one identified the lack of awareness to the program's expectations. Each teacher had their own expectations within the classroom. Teachers are working diligently and helping children reach grade-level reading. A major finding was the lack of adherence to the reading program. Teacher 1 stated, "Minimal adherence on the part, the expectations were not defined, and it was here it is, go for it." Teacher 4 iterated teacher 1 stating:

I followed the expectations about $70 \%$, I adhered to the program to some degree. I believe if I was more comfortable with the reading program, I would have adhered to the reading program's expectations and would be more successful.

These findings indicated that teachers are not being held accountable to the reading program's expectations. The lack of accountability is directly related to the lack of adherence. Adherence to a reading program is justified with a checklist or a log that tracks implemented strategies. Logs and checklist provide teachers and administration the opportunity to hold each other accountable. Throughout the interviews, teachers failed to mention the use of a checklist, a log, or any evidence that justifies adherence to the reading program. Kim et al. (2016) suggest using a checklist to keep track of the core components used during the reading program. A checklist helps teacher adhere to the reading programs expectations. Also, a checklist can help teachers identify deviations from the program's expectations.

To achieve adherence, the school must consider classroom management support. A successful teacher must have ways to deal with classroom management issues positively. The lack of classroom management can deplete valuable instructional time (Phillips, Ingrole, Burris, \& Tabula, 2017). Dealing with classroom management issues compounds the obstacles teachers must overcome during the implementation process. Time invested in dealing with behavioral issues can affect the program's outcome. Holding teachers and stakeholders accountable to meet program goals was essential in this investigation. Overall the reading program expectations were not defined clearly. Teachers were given a reading curriculum with an expectation to deliver a reading program. Adherence was not measured and a lack of evidence indicating a tool to measure adherence.

\section{Theme 2: Insufficient Amount of Dosage (Teacher Dosage)}

Theme two points to the lack of teacher training and support. The lack of training and support was evident throughout the investigation. Question number four of the interview protocol focuses on dosage received by teachers. The amount of training, coaching and support teachers received during the reading program represents dosage in the study. All six teachers responded to question number four with two-hour training at the beginning of the school year. Teacher 6 indicated that, "The training was over a skype conference and lasted three hours." The implementation process is taking place, but there is a lack of documentation. Perhaps the lack of implementation is taking place because the reading program is computer-based, and a dashboard is available for teachers to monitor student progress. Teachers get caught up on student progress and forget about their own progress. Blended learning is making its ways into classrooms around the country. For a computer-based blended program to be successful four key components must be in place: 1. relevant teacher training 2. technical support 3. program effectiveness related to student outcome 4. importance of understanding the program (Kim et al., 2016). Components 1,3 , and four are directly related to adherence and dosage. More importantly, the four components mentioned in Kim et al. (2016) study were not demonstrated in the investigation and played a significant role in FOI at GS. Teacher 3 responded to question 2 as follows:

I had classroom management issues that made it difficult to adhere to the program's expectation. Also, I 
had difficulties with technical support and teacher training. I remember a few incidents where a student took two days to finally $\log$ in, these were the issues I was facing. I was not effective at all.

Teacher 2 had issues with three of the four components mentioned by Kim et al. (2016). Effective teacher training incorporates the right amount of dosage and provides ways to monitor the implementation of the program. In this study, a small amount of evidence of teacher dose is present. According to Schechter, Kazakoff, Bundschuh, Prescott, and Macaruso (2017), "A blended program should be more than just a computer program that a school uses to mitigate the reading program" (p. 454). At GS, the school is aware of the reading program, but several teachers indicate that more training and support is needed. A two-hour crash course is not enough for teachers to implement a reading program with high fidelity.

The school implements the reading program as their primary curriculum; therefore, most of the curriculum derives from their reading program. Data from this investigation reveals that monitoring and tracking the time a teacher is involved in the reading program is missing. Teacher 4 stated that, "A lack of dosage at the school was evident." Teacher 4 goes on to say that, "Teachers did not receive adequate training to implement this reading program properly." Babinsky, Amendum, Knotek, Sanchez and Malone (2018) consider, "The support of teachers through high impact instructional strategies, the use of mentors/coaches, and focused teacher training" (p. 119). The high-impact instructional strategies are geared towards the development of phonemic awareness, phonics knowledge, and segmenting. The three high-impact strategies require ongoing support for teachers. Mentoring is effective if it takes place routinely throughout the year (Babinsky et al., 2018, p.120). An effective program implementation requires a sustained mentoring program throughout the year; Moreover, the mentoring/coaching program needs to focus on student achievement. A program achieving student success is a program that meets the intended outcome.

According to the findings, teachers received a 2-hour dose of training. The training was via Skype, and the goal was to provide a foundation of the reading program. The reading program took place all year, but there was a lack of follow-up. The teachers finished the school year without any closure, or any form of evaluation from the reading program's representatives. The structure (adherence and dosage) of the reading program at GS has room for improvement.

\section{Research Question 2}

Research Question 2 asked the following: What constitutes the process of the reading intervention curriculum at GS? The goal of this research question was to explore the change made to the reading program with respect to what is already in place at GS. Also, to highlight the quality of instruction presented by the six participants at GS. Responses to RQ 2 identified four themes. The four themes included the extent of differentiation, pacing plan, unalignment of differentiation strategies to the reading expectations and the identification of quality of instruction in the reading program.

\section{Theme 3: Different Approaches to Differentiation}

Theme three highlighted teacher differentiation at GS. The process of implementation revealed some evidence of differentiation. For the most part, the reading program was making most of the changes to the curriculum. A few teachers took differentiation to another level. Teachers that took differentiation to another level had the opportunity to spend time with students and further differentiate instruction. According to Dijkstra, Walraven, Mooij and Kirschner (2016), "Differentiation should be part of a reading program" (p. 154). Differentiation allows teachers to spend valuable instructional time with struggling learners. Teacher take the time to explain the reading task in a way in which each learner can comprehend. Four teachers mentioned the differentiation process; however, each teacher had a unique perspective of differentiation. Teacher 1 referred to differentiation and stated that "she was afraid to differentiate." Her fears came from differentiating to the point that a change in the reading program might occur. Dijkstra et al. (2016) argue that differentiating instruction in an intervention program is challenging to accomplish, because teachers are not fully trained to change or modify the curriculum to fit every learning style. A teacher with more experience and comfort with a reading program would be able to differentiate instruction with less difficulty. Teacher 4 illustrates this point, she claims that, "Her expertise allows her to differentiate." She goes on to say, she felt comfortable differentiating the curriculum, but was not sure if the change would alter the curriculum. Approximately $50 \%$ of the teachers revealed that the program differentiated instruction for each learner, and they did not have to differentiate instruction. A few teachers took differentiation to another level. They had the opportunity to spend time with students and further differentiate instruction. Teachers had time to conference with students so that, teachers can identify existing reading issues in the classroom. 


\section{Theme 4: Issues with the Pacing Plan}

Theme 4 brings to light the lack of a pacing plan to deal with the rigidity of the reading program. Students take a pre-assessment reading test; following the test, students are placed at a reading level. Once placed, the reading program differentiates instruction to fit the learner's reading need. However, data indicates that adaptation to the reading program are difficult. The program is rigid; therefore, making differentiating a challenge for a teacher. A program that lacks a pacing guide may have a difficult time making adjustment throughout the process; as a result, leading to unfavorable instructional decisions. Often a lack of training can lead to bias and assumptions made by the program. The program can assume that the intervention did not work and abundant the reading intervention program. Evidence points to the, "struggles" teachers experienced to keep up with the intended program. Teacher 4 states that "I had no pacing guide, I had to use the own, in fact I used another school district's reading curriculum and pacing guide." Teacher 5 indicates that she worked with another teacher to develop a pacing plan that they did not finish. Teacher 6 discusses her perspective on the issues she had with the pacing plan:

I had the opportunity to develop the own pacing guide. Creating the pacing plan was a challenge because I do not have experience developing a pacing plan. Without a pacing plan it was hard to make changes to the reading curriculum. I wanted to keep up with the other teachers. I guess I did not know how to make modifications, and I was afraid to change the curriculum because I could jeopardize the quality of the reading program.

The lack of a pacing plan and the rigidity of the program makes it problematic to meet program expectations. Most teachers used their expertise to deliver the reading program. Teacher 5 states that "she is currently working with a university to implement a pacing plan." A university is going to partner up with the school to help in a few parts of their reading program.

\section{Theme 5: Identifying the Quality of Instruction in the Reading Program}

Theme 5 revealed the different perceptions teachers had on quality of instruction. Another critical aspect of the implementation process is the quality of teaching. In the present study, quality of teaching consists of evidence-based instructional strategies and instructional decision making. Beecher, Abbott, Peterson and Greenwood (2017) claim that "the quality of teaching can be amplified if properly monitored through a checklist" (p. 600). In their study, Beecher et al. refer to a checklist as the quality of literacy implementation checklist. This checklist measures the overall quality of a reading program. The checklist focuses on teacher behavior, student behavior, differentiation of instruction, and the opportunity to be evaluated by their peers (teachers, coaches, and administrators). The components as mentioned above, can be considered as parts of the quality of instruction. In the case of GS, a checklist would help them understand the meaning of quality of instruction. Here are some responses by teachers regarding quality of teaching. Teacher 1 stated, "We use what we have in our teacher toolbox of strategies." Teacher 2 refers to anchor charts to represent evidence-based instructional strategies that lead to quality of instruction. Teacher 3 discusses her perception of evidence-based instructional strategies:

I had an opportunity to teach a cool reading lesson where I used cross-curricular strategies to help the students. I had students read an article on ocean ecosystems and I merged a social studies lesson to the reading program. I actually had a chance to apply for this field trip scholarship to take the students to the Channel Islands, California. We did this entire lesson using the reading the program and the kids got to learn about the ecosystems that exist in on the Channel Islands.

Teacher 5 had her unique perspective on evidence-based instructional strategies that lead to quality of instruction. She states that "there is a need for uniformity during teaching time, we (teachers) must all be using the same teaching strategies." The term uniformity refers to the fact that a few strategies are being selected and every teacher will implement the same strategies. Teacher 5 indicates a working relationship with a local university next school year, to help the school implement instructional strategies that work for all learners. She further elaborates on the partnership with the university and says, "I get a chance to implement these strategies and see if they are compatible with the reading programs expectations." A follow-up question regarding instructional strategies that lack alignment with the programs reading expectations was asked. Teacher 5 explains in the following manner, "We decide as a department to make changes to the strategy or to we get rid of it." Teachers all agree that they have autonothe in the reading program. If changes occur, teachers have the authority to make these changes. Teachers are using their prior knowledge to guide them during the implementation process. According to Knowles (1984) successful programs allow a teacher to use their prior knowledge during the implementation process. The use of prior knowledge is a component of andragogy, and andragogy is important during the adult learner's participation in an event. In this case, the event is the reading program. 
A question was asked form the interview protocol regarding Knowles theory of adults learning through a problem-solving mechanism. All six teachers had conflicting responses to a question regarding the program being problem centered. Knowles (1984) predicted that a problem centered event could lead to an adult learner being successful in that event. One of 6 teachers indicated that the program was problem centered. She referred to the reading problem as the problem centered component. The other 5 had mixed ideas about the meaning of problem centered. Teacher 3 stated that, "The reading program was more cyclical." From the researcher's understanding, cyclical was referred to one step, the next step and then every step repeats itself. Teacher 1 states "I don't think it was problem centered because students came in with different reading levels and we had to fix the problem." She continues with the following response "I would like to see a method to identify something as being problem-centered and then follow some sort of protocol/method to solve the problem, a model if you will."

\section{Research Question 3}

Research Question 3 asked the following question: What are the teacher's perceptions of the reading curriculum at GS? Questions 14 and 15 from the interview protocol were used to answer RQ 3. The intent of the of RQ 3 was to elicit responses that will link teacher responsiveness to a positive perception of the program. The goal was to get teachers to discuss their responsiveness to the program's expectations. Question 14 deals with teachers reflecting on their teaching practice during the delivery of their reading program. An assumption was made by the researcher, that is the more teachers reflect the more responsive teachers would be to the reading program. Question 15 concentrates on teacher's overall perception of the reading program. One theme was derived from RQ 3.

\section{Theme 6: Willingness to Make a Difference}

Theme 6 focused on linking teacher's perception of the reading program to teacher responsiveness. A teacher's perception was favorable if the level of responsiveness was high. Question 15 from the interview protocol allowed teachers to share their overall perception of the reading program. More training, and room for improvement was the overall perception of the teachers at GS. Two of the six teachers suggested more training. Teacher 2 enjoyed the way the program differentiates instruction for each reader. However, she mentions that,"If we are to see positive results in reading, more training is needed throughout the year." More training resonates throughout the teacher participants at GS. Teacher 6 is adamant about further teacher training and support. Teacher 1 states, "it was ok, could have been better." Following up with teacher 1 was necessary because more details were needed regarding question 15 . The follow-up question was regarding how the program can improve. She mentioned more training and sustained support throughout the year. Also, she explains that "a set curriculum that compliments the reading program at GS." Teacher 4 states that "improvement is needed and teachers must stick to a pacing plan that would be developed by the teachers. Question 8 of the interview protocol focuses on the adaptability of the program. Three out of six teachers mentioned the need to stick to a pacing plan and have adequate support to make a significant adjustment to the pacing plan. Meaningful changes in this context refer to more teacher training.

Teachers want to do better; in fact, every teacher participant reflects upon their craft (especially during the reading program). Findings indicated that the reading program needs to provide training and time for teachers to reflect on their work. Teacher participants were taking the time to reflect on their teaching practices because their credentialing program requires them to reflect (teacher 2, and 4). One teacher indicated that, "Reflecting is part of our culture here at school." Teacher 3 reflected twice a week. Reflection is part of the school's practice, but a lack of evidence was present. Every teacher seemed to be reflecting in their way and on their schedule. Overall the perception at GS was positive. These findings indicated that teachers were willing to work hard and positively respond to the reading program.

Teacher responsiveness in an essential component of FOI. In the present study, teachers responded average to the overall program. According to Woulfin (2015), "Teacher responsiveness to a reading program increases based on the urgency a school has on that part of a reading program" (p. 549). The more emphasis put on a component by an external force (administrator, district, and state) the more that component will be utilized. In the case of the GS, the school focuses on the reading program's ability to differentiate; therefore, differentiating was a leading component of the reading program. Again, teachers will respond positively if the reading program's infrastructure is held together with strong reading pillars.

\section{Discussion}

This research stems from experience of implementing programs that did not work. The lack of support and guidance indicated that it was not the program that did not work, it was a broken system that needed support. District leaders 
must do more than blindly lead a teacher to fail. Through research, it was apparent that there needs to be guidance for teacher success. There ia a gap in practice by districts leaders that support this problem. The problem was at a small school that struggled with the implementation of a reading program.

This research was grounded on Knowles' theory of Andragogy (Knowles 1984). The research design used in this study was a qualitative case study and three research questions guided this study. The collection of data was made possible via a semi-structured interview of six teachers. Emerging themes were made possible by the organization of data and the process of coding. Research findings revealed several factors affecting the current reading program. The positive social change that this study has on academia is the positive impact it has on teachers to deliver a successful reading program. Successful implementation of reading programs will lead to more advanced and literate nation.

A study conducted by Kim et al. (2016) points to the need for alignment of the curriculum to assessment outcomes (p. 365). Teachers and students are given the daunting task to perform at high levels of expectation using an unaligned process. Teachers are told to deliver a reading program with minimal training and students are expected to reach proficiency with an unaligned curriculum.

Teachers and students must be aware of the learning outcomes before a program is delivered. According to Kratz et al. (2018) the higher the implementation climate, the greater the student outcome becomes. In this context implementation climate refers to the extent to which use of an intervention is expected, supported, and rewarded. That is, curricular awareness increases the overall success of the program. Also, the implementation climate and implementation fidelity are directly related because both have a set of expectations. Implementation climate can be set in the classroom through professional development or teacher training. The teacher training program can outline the program's expectations and help teachers monitor their own progress and student progress. Student learning outcome can be enhanced by proving a reading program that is relevant, accessible, engaging and cognitively challenging (Kim et al., 2016). A reading program that is engaging provides students with the opportunity to practice in the classroom. An engaging classroom is a classroom with a high level of confidence. An accessible reading program aligns a learner's reading ability with Lexile level. Differentiating instruction for students provides a steppingstone for students to reach proficiency.

Another concern at GS is the deviation from the intended programs expectations. Teachers at GS implemented a reading program with a lack of uniformity. To develop a program that will address this concern literature was reviewed on implementation fidelity assessment. According to Smith et al. (2019) to ensure teachers are implementing the intended program "with high fidelity data must be collected to assess the level of fidelity" (p. 73). Data collection on fidelity can be used to modify the curriculum without jeopardizing the integrity of the reading program. Also, assessing implementation fidelity allows teachers to understand the reasons why a program succeeded or failed. Smith et al. suggest integrating fidelity data with student learning outcomes and using assessment data to understand the unknown features that lead to the failure of a reading program. Unknown features in this context are unexplained reasons why a program fails to deliver positive results. Some unknown variables can include: modifications that alter the program's integrity, not completing the delivery of the program as it was intended, and the role a teacher plays during the implementation process.

Learning targets, and assessment cycles are important to the implementation of a program. The two constructs can provide a roadmap for curriculum builders. Implementation fidelity can be ensured by using a checklist. A checklist brings a few important checkpoints to the implementation fidelity process. This section of the review of literature provides details about the benefits of a fidelity checklist. According to Swain, Finney, and Gerstner (2013) rarely is the alignment of the planned intervention and the implemented intervention assessed simultaneously. The entire review of literature up to this moment has mentioned the need to align a curriculum with the actual outcome of the program. A dearth of evidence prevails in academia regarding instrumentation used to assess implementation fidelity.

\section{Conclusions}

The findings of this research study opens the doors to explore possibilities for further research. One possibility is to create intervention programs that are implemented using a fidelity checklist, and classroom observations. Together, these two elements can provide a greater impact on reading achievement because the fidelity aspect of the program can provide the framework for the intervention. Also, the observations can provide greater validity to the reading program by triangulating data collection during the delivery of the program. Salient data reveals that teacher support is paramount to the success of a reading program. Also, a reading program must provide sustained support for teachers. If these two variables are present, there is a higher chance of implementation fidelity. 


\section{References}

Andrews-Larson, C., Wilson, J., \& Larbi-Cherif, A. (2017). Instructional improvement and teachers' collaborative conversations: The role of focus and facilitation. Teachers College Record, 119(020301), 1-37.

Babinski, L. M., Amendum, S. J., Knotck, S. E., Sanchez, M., \& Malone, P. (2018). Improving young English learners' language and literacy skills through teacher professional development: A controlled trial. American Educational Research Center, 55(1), 117-143. https://doi.org/10.3102/0002831217732336

Beecher, C. C., Abbott, M. I., Peterson, S., \& Greenwood, C. R. (2017). Using the quality of literacy implementation checklist to improve preschool literacy instruction. Early Childhood Educational Journal, 45, 595-602. https://doi.org/10.1007/s10643-016-0816-8

Buthane, K. S. (2017). Effects of coaching on teachers' implementation of tier I school-wide positive behavioral interventions and support strategies. Journal of Positive Behavior Intervention, 19(3), 131-142. https://doi.org/10.1177/1098300716680095

California Assessment of Student Performance and Progress. (2018a). Smarter balanced assessment result for: Los Angeles Unified. Retrieved from https://caaspp.cde.ca.gov/

California Assessment of Student Performance and Progress. (2018b). Test results for English language arts/literacy. Retrieved from https://caaspp.cde.ca.gov/

Creswell, J. W. (2012). Educational research: Planning, conducting and evaluating qualitative and quantitative research (4th ed.). Boston, MA: Pearson.

Dane, A. V., \& Schneider, B. H. (1998). Program integrity in primary and early secondary prevention: Are implementation effects out of control? Clinical Psychology Review, 18(1), 23-45. https://doi.org/10.1016/s0272-7358(97)00043-3

De La Paz, S., Felton, M., Monte-Sano, C., Croninger, R., Jackson, C., Deogracias, J. S., \& Polk-Hoffman, B. (2014). Developing historical reading and writing with adolescent readers: Effects on student learning. Theory \& Research in Social Education, 42, 228-274. https://doi.org/10.1080/00933104.2014.908754

Dijkstra, E. M., Walraven, A., Mooij, T., \& Kirschner, P. A. (2016). Factors affecting intervention fidelity of differentiated instruction in kindergarten. Research Papers in Education, 32(2), 151-169. https://doi.org/10.1080/02671522.2016.1158856

Finney, S. J. (2019). Using Implementation fidelity data to evaluate \& improve program effectiveness [PowerPoint slides]. Retrieved from http://jmu.edu/assessment/sass

Fogarty, M., Oslund, E., Simmons, D., Davis, J., Simmons, L., Anderson, L., Clemens, N., \& Roberts, G. (2014). Examining the effectiveness of a multicomponent reading comprehension intervention in middle school: A focus on treatment fidelity. Education Psychology Review, 26, 425-449. https://doi.org/10.1007/s10648-014-9270-6

Guo, Y., Dynia, J. M., Logan, J. A. R., Justice, L. M., Breit-Smith, A., \& Kaderavek, J. N. (2016). Fidelity of implementation for an early - literacy intervention: Dimensionality and contribution to children's intervention outcomes. Early Childhood Research Quarterly, 37(1), 165-174. https://doi.org/10.1016/j.ecresq.2016.06001

Hall, W. J., \& Chapman, M. V. (2018). Fidelity of implementation of a state antibullying policy with a focus on protected social classes. Journal of School Violence, 17(1), 58-73. https://doi.org/10.1080/15388220.2016.1208571

Kaimal, G., \& Jordan, W. J. (2016). Do incentive-based programs improve teacher quality and student achievement? An analysis of implementation in 12 urban charter schools. Teacher College Record, 118, 1-34.

Kim, J. S., Hemphill, L., Thomson, J. M., Jones, S. M., LaRusso, M. D., \& Donovan, S. (2016). Engaging struggling adolescent readers to improve reading skills. Reading Research Quarterly, 52(3), 357-382. https://doi.org/10.1002/rrq.171

Knowles, M. S., Holton, E. F., \& Swanson, R. A. (1973). The adult learner: The definitive classic in adult education and human resource development. Burlington, MA: Butterworth-Heinemann.

Kratz, H. E., Xie, M., Marcus, S. C., Stahmer, A., Locke, J., Beidas, R., \& Mandell, D. S. (2019). The effect of implementation climate on program fidelity and student outcomes in autism support classroom. Journal of Counseling and Clinical Psychology, 87(3), 270-281. https://doi.org/10.1037/ccp0000368 
Lambert, R., Gallagher, P. A., \& Abbott-Shim, M. (2015). An evaluation of the intensity of mentoring: Child outcomes. Early Child Development and Care, 185(8), 1314-1330. https://doi.org/10.1080/03004430.2014.992426

Little, M. A., Riggs, N. R., Shin, H. S., Tate, E. B., \& Pentz, M. A. (2015). The effects of teacher fidelity of implementation of pathways to health on student outcomes. Evaluation \& The Health Professions, 38(1), 21-41. https://doi.org/10.1177/0163278713489879

Lodico, M. G., Spaulding, D. T., \& Voegtle, K. H. (2010). Methods in educational research: From theory to practice. San Francisco, CA: Jossey-Bass.

Meriam, S. B., \& Tisdell, E. J. (2016). Qualitative research: A guide to design and implement. San Francisco, CA: Jossey-Bass.

Moore, A. M., Gove, A., \& Tietjen, K. (2017). Great expectations: A framework for assessing and understanding key factors affecting student learning of foundational reading skills. New Direction for Child and Adolescent Development, 155(1), 13-30. https://doi.org/10.1002/cad.20192

O'Reilly, T., Weeks, J., Sabatini, J., Halderman, L., \& Steinberg, J. (2014). Designing reading comprehension assessments for reading intervention: How a theoretically motivated assessment can serve as an outcome measure. Educational Psychology Review, 26(1), 403-424. https://doi.org/10.1007/s10648-014-9269-z

Phillips, B. M., Ingrole, S. A., Burris, P. W., \& Tabula, G. (2017). Investigating predictors of fidelity of implementation for a preschool vocabulary and language curriculum. Early Child Development and Care, 187(3), 542-553. https://doi.org/10.1080/03004430.2016.1251428.

Polkinghorne, F. W. (2013). Integrated reading literacy intervention (irlis): A mixed-method analysis of the perceived characteristics for effective training. The Journal of Research in Business Education, 55(2), 36-49.

Smith, K. L., Finney, S. J., \& Fulcher, K. H. (2019). Actionable steps for engaging assessment practitioners and faculty in implementation research. Research Practice in Assessment, 1, 71-81.

Suhrheinrich, J., \& Chan, J. (2017). Exploring the effect of immediate video feedback on coaching. Journal of special Education Technology, 32(1), 47-53. https://doi.org/10.1177/0162643416681163

Swain, M. S., Finney, S. J., \& Gerstner, J. J. (2013). A practical approach to assessing implementation fidelity. Assessment Update, 25(1), 5-7, 13.

Taylor, B., \& Kroth, M. (2009). Andragogy's transition into the future: Meta-analysis of andragogy and its search for a measurable instrument. Journal of Adult Education, 38(1), 1-11.

Uzair-ul-Hassan, M., Parveen, I., \& Riaz, M. (2016). Active and receptive behaviors of trainee teachers and students during teaching-learning process in classroom. Journal of Educational Research, 19(2), 15-29.

Van Kuijk, M. F., Deunk, M. I., Bosker, R. J., \& Ritzema, E. S. (2015). Goals, data use and instruction: The effect of a teacher training program on reading achievement. An International Journal of Research, Policy and Practice, 27(2), 135-156. https://doi.org/10.1080/09243453.2015.1026268

Vollmer, L. E., Gettinger, M., \& Begeny, J. C. (2019). Training preservice general teachers in response to intervention: A survey of teacher educators throughout the United States. Journal of Applied School Psychology, 35(2), 122-145. https://doi.org/10.1089/15377903.2018.1528488

Wlodarsky, R. (2018). The benefits of reflection on improving teaching through change: A reflective model for professional development. National Education Journal, 11(1), 33-41.

Woulfin, S. L. (2015). Highway to reform: The coupling of district reading policy and instructional practice. Journal of Educational Change, 16, 535-557. https://doi.org/10.1007/10833-015-9261-5 